\title{
REDESCRIPCIÓN DE Bournonia gardonica (TOUCAS, 1907) (RADIOLITIDAE, BIVALVIA) Y ANÁLISIS DE LAS FACIES EN QUE APARECE (CONIACIENSE, SISTEMA CENTRAL, ESPAÑA)
}

\author{
Javier GIL', Josep Maria PONS ${ }^{2}$ y \\ Manuel SEGURA ${ }^{\prime}$
}
' Unidad de Estratigrafía. Departamento de Geología. Universidad de Alcalá. 28870) Alcalá de Henares: Madrid. javier.gil@uah.es
- Unitat de Paleontologia. Departament de Geologia. Universitat Autonoma de Barcelona. (08193 Bellaterra; Barcelona. josepmaria.pons@uab.es

Gil, J., Pons, J.M. y Segura, M. 2002. Redescripción de Bournonia gardonica (Toucas. 1907) (Radiolitidae. Bivalvia) y análisis de las facies en que aparece (Coniaciense, Sistema Central, España). [Redescription of Bournonia gardonica (Toucas, 1907) (Radiolitidae, Bivalvia) and analysis of the facies where it appears (Coniacian, Sistema Central, Spain).] Revista Española de Paleontología, 17(2), 245-256. ISSN 0213-6937.

\begin{abstract}
At the southern margin of the Sistema Central (Spain), the Cretaceous succession consists of a terrigenous lower unit (Arenas de Utrillas Formation) and a carbonate upper unit (Dolomías del Pantano de la Tranquera, Calizas de Hortezuelos, Calcarenitas de Hontoria del Pinar and Calizas del Burgo de Osma formations). Dating of the succession is problematical because of the scarcity of fossils, due to its terrigenous or dolomitic nature. The Calizas de Hortezuelos Formation, in the Barranco de las Cuevas section, presents a bioclastic upper part with little dolomitisation whose facies belong shallowing upwards sequences in a peritidal carbonate platform. One of these facies contains well preserved rudists, that have been identified as radiolitids belonging to the species Bournonia gardonica (Toucas, 1907), characteristic for the Coniacian. Their study completes the original description of the species, mainly based on external characters, particularly with regard to the shell structure. Moreover, this paper provides the first precise dating of the upper carbonate unit of the Cretaceous in all the southern margin of the Sistema Central and confirms part of the previous chronostratigraphic inferences.
\end{abstract}

Keywords: Rudists, Radiolitidae, Bournonia gardonica, Coniacian, Sistema Central, Madrid.

\section{RESUMEN}

La serie sedimentaria cretácica del borde sur del Sistema Central (España) se organiza en una unidad inferior terrígena (Formación Arenas de Utrillas) y otra superior carbonatada (formaciones Dolomías del Pantano de la Tranquera, Calizas de Hortezuelos, Calcarenitas de Hontoria del Pinar y Calizas del Burgo de Osma) y presenta problemas de datación, al ser poco fosilífera debido a su naturaleza predominantemente terrígena y dolomítica. En la sección del Barranco de las Cuevas, la Formación Calizas de Hortezuelos presenta un conjunto bioclástico superior, poco dolomitizado, cuyas facies se organizan en secuencias de somerización en ambientes perimeareales de una plataforma carbonatada. Una de estas facies contiene rudistas bien conservados, que han sido determinados como radiolítidos de la especie Bournonia gardonica (Toucas, 1907), característica del Coniaciense. Su estudio permite ampliar la descripción original de la especie, basada principalmente en caracteres externos, especialmente en la estructura de la concha. Además, este trabajo constituye la primera datación precisa de la unidad carbonatada superior del Cretácico en todo el borde meridional del Sistema Central y confirma parte de las atribuciones cronoestratigráficas realizadas con anterioridad.

Palabras clave: Rudistas, Radiolitidae, Bournonia gardonica, Coniaciense, Sistema Central, Madrid. 

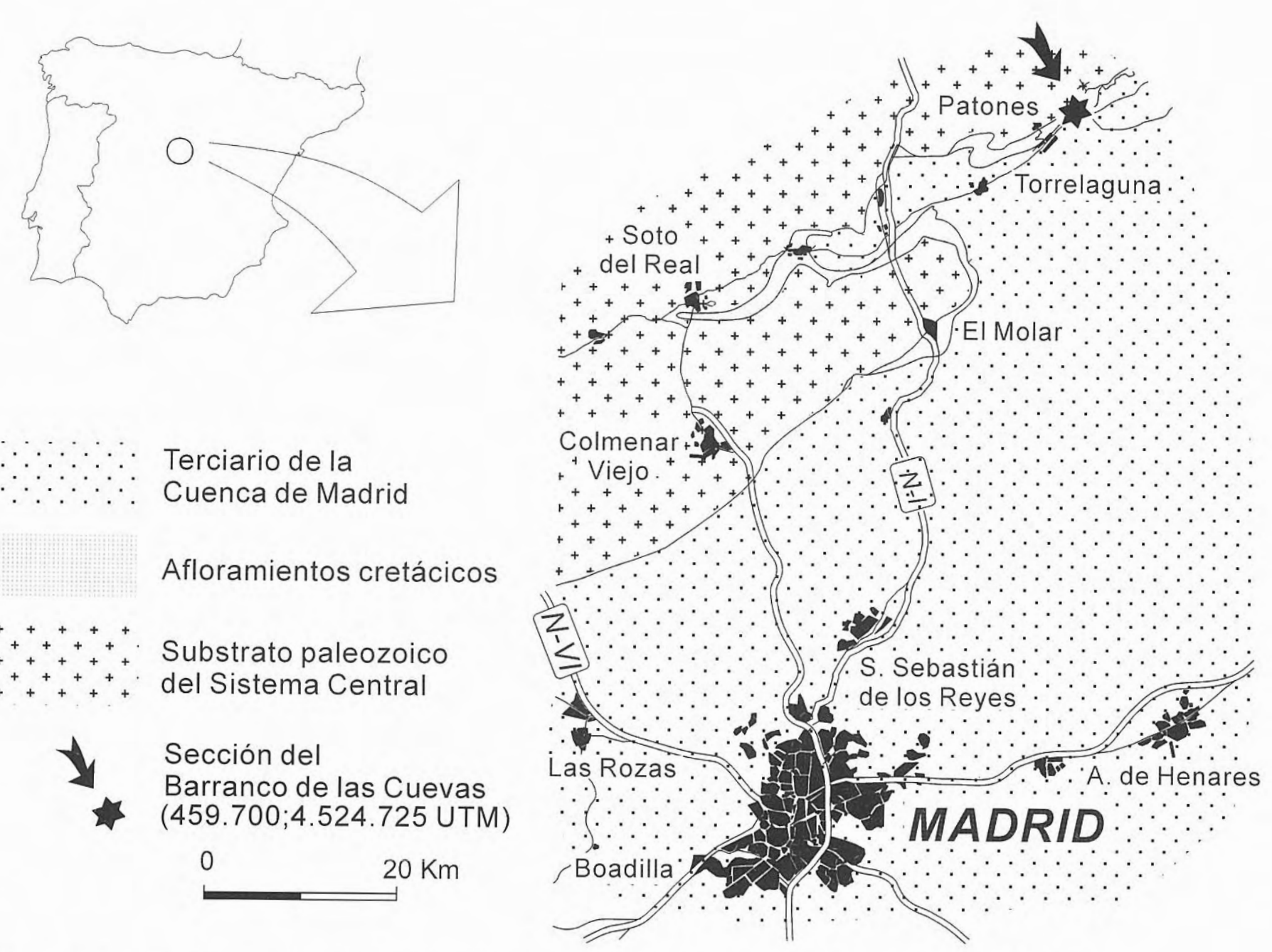

Figura 1. Localización geográfica y geológica del afloramiento del Barranco de las Cuevas (Patones, Madrid).

Geographic and geologic setting of Barranco de las Cuevas section (Patones, Madrid).

\section{INTRODUCCIÓN}

El Sistema Central constituye un rejuvenecimiento alpino de rocas ígneas y metamórficas de edad paleozoica; en sus márgenes septentrional y meridional aflora una cobertera mesozoica, constituida básicamente por depósitos cretácicos que constituyen las terminaciones hacia el Macizo Hespérico de las plataformas carbonatadas desarrolladas en zonas centrales de la Cuenca Ibérica (hoy día, Cordillera Ibérica). Las rocas del Cretácico que afloran en el borde sur del Sistema Central (Fig. 1) son en general muy poco fosilíferas, debido a su naturaleza predominantemente terrígena y dolomítica. Los únicos fósiles que hasta ahora han sido determinados con precisión son: a) los restos de coníferas, cicadales, licopodiales, filicales y angiospermas monocotiledóneas y dicotiledóneas encontrados dentro de las intercalaciones margosas del conjunto carbonatado superior en las canteras de Guadalix de la Sierra y en el área de Torrelaguna-Patones (Almendros et al., 1982; Gómez Porter, 1984; Diéguez, 1986; Álvarez Ramis y Fernández Marrón, 1990; Álvarez Ramis et al., 1992, Diéguez et al., 1993; Álvarez Ramis et al., 1996); b) el polen localizado en esta misma zona y en los mismos niveles estratigráficos (Álvarez Ramis y Doubinger, 1980; Álvarez Ramis et al., 1987). Estos fósiles han permitido identificar con escasa precisión la existencia de un Coniaciense-Santoniense, dentro de la unidad carbonatada superior de la sucesión estratigráfica.

En otros trabajos se cita también la presencia de bivalvos y fragmentos de plantas (de la Concha, 1953) y varias asociaciones de foraminíferos (Fuster y Febrel, 1958; Corchón, 1976) de los que no se han realizado determinaciones precisas. Por último, existen trabajos antiguos, como el de Prado (1864), en los que se describe una variada fauna fósil marina repartida en los diferentes afloramientos cretácicos de la Comunidad de Madrid, reconociendo braquiópodos, equinodermos, gasterópodos, algas, ostreidos y otros bivalvos, cuya clasificación no ha sido aún actualizada. Cabe destacar que este autor señala por primera vez la presencia de rudistas en los afloramientos de San Agustín de Guadalix y del Valle de Lozoya en una posición estratigráfica similar a la de los ejemplares que nos ocupan en este trabajo.

Esta escasez de fósiles tiene, entre otras consecuencias, una importante indeterminación cronoestratigráfica para las sucesiones de este margen del 


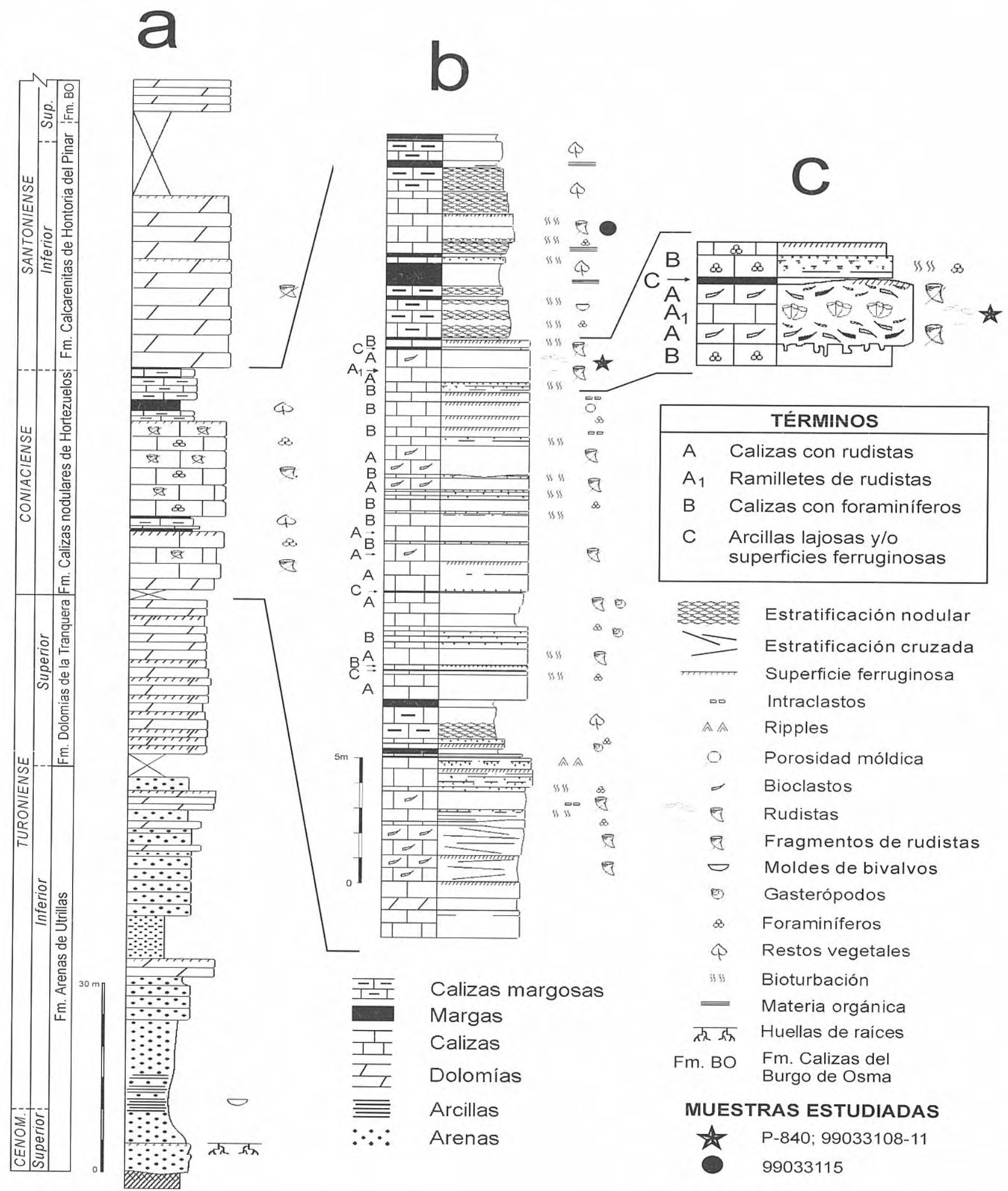

Figura 2. a. Columna estratigráfica general del Cretácico superior del Barranco de las Cuevas (Madrid) mostrando las unidades litoestratigráficas y la edad de los depósitos. b. Sección estratigráfica de detalle de la Formación Calizas de Hortezuelos, mostrando el patrón de relleno sedimentario en el banco carbonatado intermedio. c. Términos elementales y asociación de facies en el tramo con los rudistas estudiados.

a. Upper Cretaceous stratigraphic profile at Barranco de las Cuevas (Madrid) showing litostratigraphical units and chronostratigraphy. b. Detailed stratigraphic section of the Hortezuelos Limestone Formation. The sedimentary stacking pattern of the carbonate bundle with rudists is shown. c. Elemental terms and facies association in the studied rudist beds. 
Sistema Central. Hasta ahora, las dataciones se han realizando atribuyendo a las unidades del Cretácico una edad en función de su posición estratigráfica o por correlación con las unidades litoestratigráficas definidas en su borde norte (Alonso y Mas, 1982), tal y como establecen Bellido et al. $(1987,1988)$ y Portero et al. (1990) en los mapas geológicos de la serie MAGNA a escala 1:50.000 de Valdepeñas de la Sierra, Torrelaguna y Buitrago de Lozoya, entre otros.

En los últimos años se viene realizando en el borde meridional del Sistema Central un análisis estratigráfico de detalle en el que se han identificado las grandes discontinuidades estratigráficas de la sucesión sedimentaria, que limitan secuencias deposicionales o unidades de roca genéticamente relacionadas. Estas secuencias deposicionales se han correlacionado con sucesiones más completas, mejor conocidas y mejor datadas de zonas internas del Surco Ibérico, así como con las cartas de ciclos eustáticos de Haq et al. (1988), lo que ha permitido realizar una atribución cronoestratigráfica más precisa de los diferentes tramos de la sucesión cretácica (Gil y García, 1996; García et al., 1996). Para dicho análisis estratigráfico ha sido determinante el estudio detallado de la sección estratigráfica del Barranco de la Cuevas, en la cual las rocas carbonatadas están sólo parcialmente dolomitizadas, habiéndose podido reconocer, en parte de la sucesión, las facies y estructuras sedimentarias originales, así como su contenido fósil.

En este trabajo se realiza un análisis de las facies y los medios sedimentarios de la Formación Calizas de Hortezuelos y se identifica, describe y discute su fauna de rudistas. Con ello, se data por primera vez la serie carbonatada del Cretácico en el área de Torrelaguna-
Patones, lo que constituye además la primera datación precisa de la unidad carbonatada superior del Cretácico en todo el borde meridional del Sistema Central y, junto con los datos bioestratigráficos anteriores, confirma parte de las atribuciones cronoestratigráficas realizadas con anterioridad (Gil y García, 1996; García et al., 1996).

Trabajos en curso confirman la presencia de rudistas en afloramientos próximos (Soto del Real, Arrebatacapas, Barranco del Mortero, Pontón de la Oliva).

\section{ESTRATIGRAFÍA}

En esta región, el Cretácico define morfológicamente un relieve en cuesta vergente al $\mathrm{NO}$, que se apoya discordante sobre una potente paleoalteración desarrollada sobre pizarras del Ordovícico. El registro estratigráfico está compuesto por una unidad inferior terrígena (Formación Arenas de Utrillas en sentido amplio) y otra superior carbonatada (Corchón, 1976); en esta última, se reconocen de muro a techo las formaciones Dolomías del Pantano de la Tranquera, Calizas de Hortezuelos, Calcarenitas de Hontoria del Pinar y Calizas del Burgo de Osma (Gil y García, 1996), estas dos últimas dolomitizadas (Fig. 2a).

En la sección del Barranco de las Cuevas, la Formación Calizas de Hortezuelos presenta un espesor de $42 \mathrm{~m}$. Su límite inferior con la Formación Dolomías del Pantano de la Tranquera se sitúa dentro de un pequeño tramo cubierto y queda puesto de manifiesto por un brusco cambio de facies; en afloramientos y sondeos próximos (Gil et al., 2000) se reconoce en un nivel de brechas dolomíticas y arcillas negras ricas en materia orgánica, que confirman la existencia de una discontinuidad estratigráfica. El límite superior se

Figura 3. a. Aspecto de campo de las calizas bioclásticas con fragmentos de rudistas (Término A). b. Ramillete de rudistas en posición de vida (Subtérmino $A_{1}$ ). c. Aspecto de campo de la alternancia de calizas micríticas con foraminíferos (Término B), en la que se reconocen estructuras fenestrales, y calizas bioclásticas (Término A), apreciándose sus diferencias de color y textura. $\mathbf{d}$. Detalle de la superficie irregular de contacto entre los dos términos, en la que destacan los intensos procesos de bioerosión a techo del Término B, previamente litificado. e. Aspecto en el campo de la superficie del thicket de rudistas muestreado (Subtérmino $\mathrm{A}_{1}$ ). f. Sección del ejemplar PUAB-43.946 cortando transversalmente varias valvas inferiores que no presentan ninguna orientación preferente de las bandas radiales y sólo esporádica y puntualmente están en contacto con las conchas vecinas soldadas. Las conchas están incluidas en una matriz carbonatada de grano fino, intensamente dolomitizada durante una diagénesis temprana (ver detalles de figuras posteriores), que también rellena sus huecos internos. Presentan señales de fractura, aunque con mínimo desplazamiento de las partes; puede interpretarse que se debe a la compactación dentro de un sedimento ya previamente consolidado. g. Vista ventral externa del ejemplar juvenil PUAB-43.932 mostrando las dos bandas radiales y la interbanda. La conservación de la banda anterior es incompleta en la parte inferior de la concha. La barra de escala, en e, f y g, corresponde a $5 \mathrm{~mm}$.

a. Aspect of bioclastic limestone with rudist fragments (Term A). b. Bouquet of rudists in life position (Subterm $A_{1}$ ). c. Aspect in the outcrop of the alternate micritic limestone with foraminifera (Term B), with fenestral fabric, and bioclastic limestone (Term A), showing differences in colour and texture. d. Detail of the stratigraphic surface between both terms, showing the intense boring processes over a previously lithified micritic sediment. e. Aspect in the field of the surface of the sampled rudist thicket (Subterm A,). f. Cross section of the specimen PUAB-43.946, transverse section of several lower valves, with no preferred orientation of the radial bands and neighbours occasionally in contact with their shells attached. Shells are included in a fine grained carbonate matrix, highly dolomitised during early diagenesis (see details in following figures), which also fills their cavities. Fracture scars, although with very little movement of parts, are evident; they can be interpreted as due to compaction in previously consolidated sediment. g. External ventral view of the juvenile specimen PUAB-43.932 showing the two radial bands and the inter-band. Anterior band is not fully preserved at the lower part of the shell. Scale bar in $\mathrm{e}, \mathrm{f}$ and $\mathrm{g}$, corresponds to $5 \mathrm{~mm}$. 

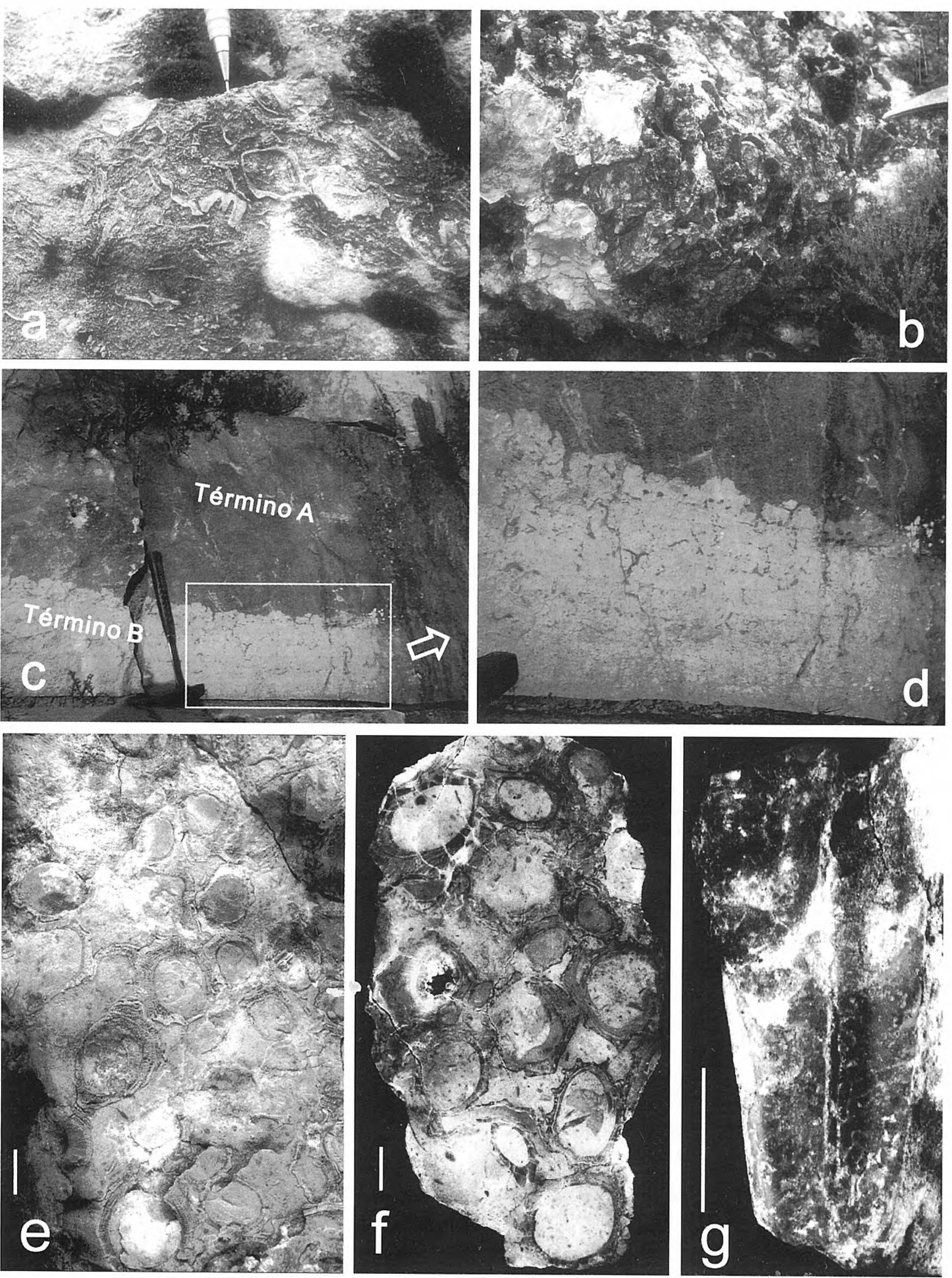
Figura 4. Bournonia gardonica (Toucas). Sección transversal en lámina delgada de la valva inferior (derecha) de uno de los escasos ejemplares aislados, PUAB-43.933. La barra de escala corresponde a $5 \mathrm{~mm}$. a. Sección completa, mostrando la banda anterior $(\mathrm{AB})$ la posterior (PB) y la interbanda (IB). La concha aparece ligeramente colapsada del lado dorsal (parte superior de la fotografía) pero muestra suficientemente la ausencia de cresta ligamentaria. b-c. Detalles de la parte anterodorsal en que se observa que las láminas pueden ser compactas o presentar crestas radiales originadas por pliegues en las mismas, se evidencia la transición de una a otra estructura y que en ambos casos la microestructura es prismática simple. Asimismo, es visible el aspecto regular de la estructura en el margen interno de la concha. d-e. Detalles de la banda posterior y de la banda anterior, respectivamente. Las bandas corresponden a pliegues planos hacia abajo y hacia fuera de las láminas. El pliegue anterior, más ancho que el posterior, está ligeramente desdoblado resultando cóncavo exteriormente; las láminas, en esta sección, presentan crestas radiales en la parte interna de la concha y estructura compacta en la externa. Únicamente la parte compacta aparece con bioperforaciones. La interbanda está reducida al surco que separa las dos bandas y corresponde a un pliegue agudo hacia arriba y hacia dentro de las láminas.

Transverse thin section of the lower (right) valve of one of the rare isolated specimens, PUAB-43.933. Scale bar corresponds to $5 \mathrm{~mm}$. a. Complete section, showing the anterior band $(\mathrm{AB})$ the posterior band $(\mathrm{PB})$ and the interband (IB). The shell is slightly collapsed at the dorsal side (upper part of the photograph) but it shows clearly enough the absence of a ligamentary crest. b-c. Details of the antero-dorsal part where it can be seen that the laminae can be compact or they can present radial crests formed by folds in them; the transition from one to the other structure is evident as is also the simple prismatic microstructure in both. Moreover, the regular pattern of the structure at the inner shell margin is visible. d-e. Details respectively of the posterior and of the anterior bands. Bands correspond to flat out-and down-folds of the laminae. The anterior fold, wider than the posterior one, is slightly bent, hence concave outwards; laminae, in this section, show radial crests at the innermost part of the shell and compact structure at the outer part. Borings appear only in the compact part. The interband is reduced to the sulcus in between the two bands and corresponds to a sharp in-and up-fold of the laminae.

sitúa al comienzo de un segundo escarpe morfológico, estando reflejado por una superficie ferruginosa y por un incremento de la dolomitización, que afecta de manera notable a las formaciones superiores.

En esta sección, la Formación Calizas de Hortezuelos está constituida por dos conjuntos de calizas sobre los que se disponen dos tramos margoso-carbonatados (Fig. 2). El conjunto inferior está formado por calizas bioclásticas con estratificación cruzada en su parte inferior y por micritas bioturbadas al techo. El conjunto superior está formado por una alternancia de calizas micríticas mudstone-wackestone con foraminíferos y calizas bioclásticas packstone con fragmentos de rudistas; la proporción de calizas bioclásticas disminuye hacia el techo. Los tramos margosos están constituidos por margas y calizas margosas con fragmentos de bivalvos y restos vegetales.

\section{Facies:}

En detalle, el análisis de facies del conjunto superior, permite reconocer tres términos elementales (Fig. $2 b, c)$.

- Termino A: Calizas con rudistas; son calizas bioclásticas, algo dolomitizadas y ferruginizadas a techo, que están estratificadas en bancos de 0,4-1,2 m de espesor, presentando una continuidad lateral de unos centenares de metros. Están constituidas por fragmentos de rudistas (Fig. 3a) que han sufrido una biodegradación local y retrabajamiento, ya que las conchas están perforadas y fragmentadas; esta fragmentación se produjo por lavado del sedimento fino en el que estaban enterrados los rudistas y colapso de los individuos sueltos, dando lugar a acumulaciones en bancos bioclásticos con texturas granosoportadas packstone $\mathrm{y}$ granoclasificación positiva. Es frecuente la presencia de intraclastos micríticos escasamente reelaborados. Hacia el techo, se ha reconocido un banco no removilizado (Subtérmino $A_{l}$ ) en el que los rudistas se encuentran en posición de vida formando pequeños ramilletes de varios individuos (Fig. 3b) o un "thicket" de ejemplares sueltos (Fig. 3e,f) semienterrados en el fango carbonatado fino.

- Término B: Calizas con foraminíferos; son calizas micríticas mudstone (ocasionalmente wackestone) muy homogéneas, dispuestas en bancos tabulares de 0,1-0,2 $\mathrm{m}$ de espesor, de base plana y techo generalmente irregular. Contienen miliólidos de concha fina, ostrácodos, discórbidos y algas verdes; algunos bancos presentan estructuras fenestrales (Fig. 3d), mientras que otros se encuentran intensamente bioturbados (Fig. $3 \mathrm{c}, \mathrm{d}$ ), especialmente a techo donde están perforados por litófagos y ferruginizados, definiendo "hardgrounds" o "superficies de omisión" en el sentido de Vera et al. (1989). Por tanto, presentan dos etapas diferenciadas de actividad biogénica; una sinsedimentaria que se realiza sobre sedimento blando (burrows), y otra posterior a una consolidación parcial del sedimento (borings). En ambos casos, los tubos están rellenos por material bioclástico procedente del siguiente episodio sedimentario (Término A). Corresponden a depósitos de ambientes costeros, de baja energía e intensa actividad orgánica, con frecuentes interrupciones sedimentarias durante las cuales se produce la consolidación parcial del sedimento, el cual es posteriormente perforado y rellenado.

- Término C: Arcillas lajosas; corresponden a delgados niveles de 0,05-0,1 $\mathrm{m}$ de espesor de arcillas beiges lajosas que se disponen a modo de interestratos o juntas a techo de los bancos bioclásticos (Término A). Sin 


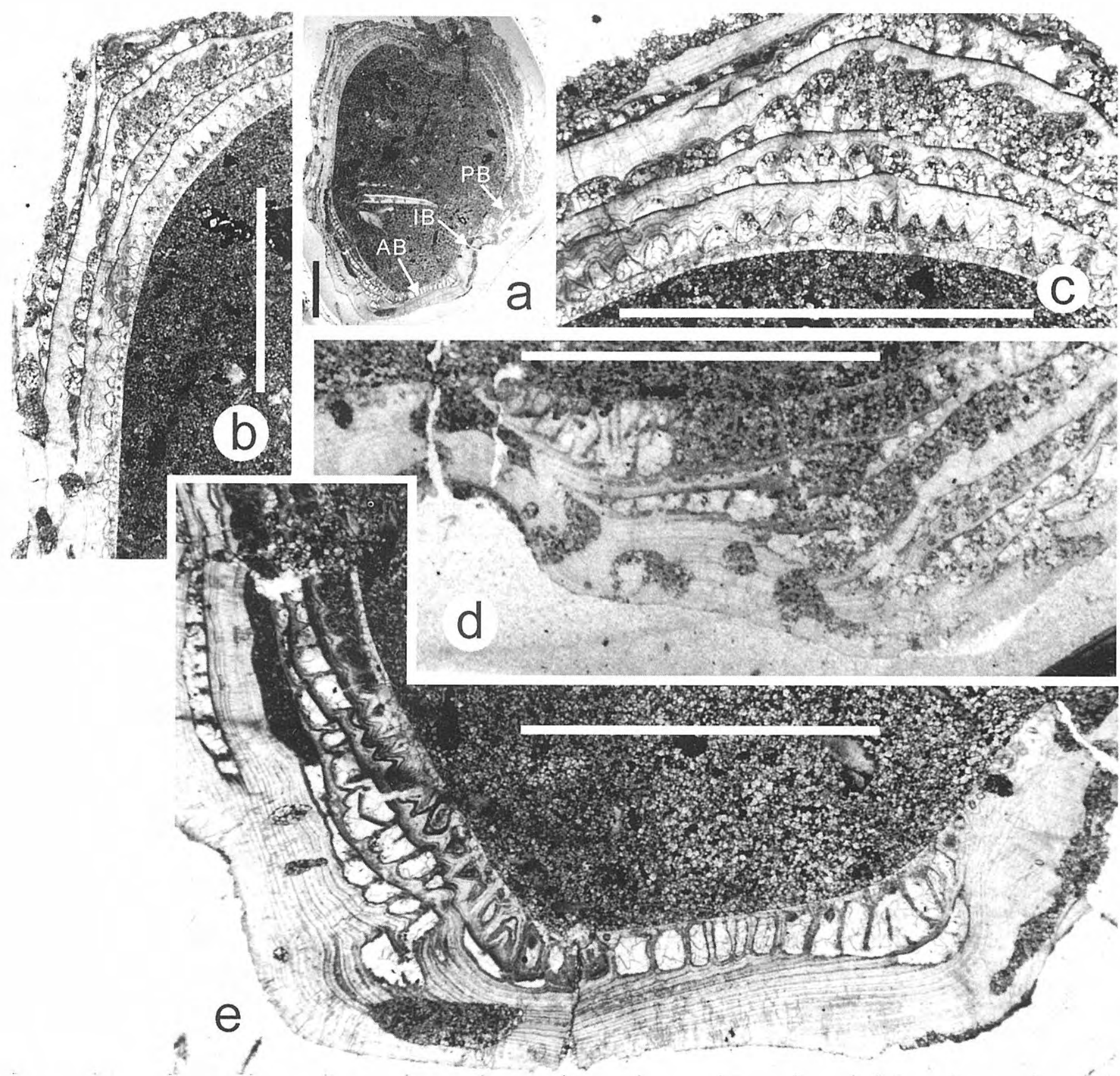

embargo, en su lugar puede aparecer una superficie ferruginosa de manera que, la presencia de una u otra puede venir dada a partir de la geometría a gran escala de los bancos bioclásticos; éstos pueden ejercer un control sedimentario de forma que sobre las crestas de las barras bioclásticas, sometidas a una mayor hidrodinámica, se desarrolle la superficie ferruginosa, mientras que en los surcos situados entre las barras se depositen las arcillas.

Estos tres términos se asocian formando una secuencia de depósito de energía decreciente (Fig. 2c); da comienzo con depósitos bioclásticos (Término A), los cuales son el resultado de episodios de tormenta durante los que se destruyen pequeños ramilletes de rudistas al lavarse el sedimento fino (fango carbonatado) que les servía de sostén; el episodio de tormenta finaliza con una ralentización o interrupción sedimentaria generándose una superficie ferruginosa o una junta de arcillas (Término C). A continuación se sucede un nuevo episodio sedimentario de bajo régimen hidráulico, durante el cual se depositan sedimentos finos carbonatados (Término B) con desarrollo de estructuras fenestrales y bioturbación; al tiempo, en sectores próximos más internos de la plataforma, se desarrollan nuevas bioconstrucciones, utilizando los niveles bioclásticos previos como soporte para la fijación de las larvas.

La secuencia sedimentaria, que se puede encontrar incompleta por la ausencia del Término B, se sucede a lo largo del intervalo estratigráfico estudiado; representa un patrón de apilamiento deposicional de naturaleza probablemente cíclica, relacionándose con secuencias de 
somerización de alta frecuencia en ambientes costeros (estructuras fenestrales) de una plataforma carbonatada (presencia de rudistas).

A escala de todo el conjunto estratigráfico, existe una gradación de muro a techo, desde depósitos bioclásticos (Término A), más abundantes en la base, a micríticos (Término B), marcando una disminución gradual en el mismo sentido del número e intensidad de etapas de tormenta. Debido probablemente a este descenso del régimen hidráulico, en la parte superior del conjunto analizado se localiza el banco con rudistas en posición de vida (Subtérmino $A_{1}$ ), incluidos dentro de un sedimento fino micrítico que rellena los huecos internos y externos, y que sufre un avanzado proceso de dolomitización temprana. Este nivel se encuentra intercalado entre facies de removilización bioclástica (Término A) dentro de una capa de 0,60 $\mathrm{m}$ de espesor y refleja unas condiciones deposicionales diferentes a las de aquellas.

\section{PALEONTOLOGÍA}

Aunque las capas con rudistas son relativamente abundantes dentro del intervalo estratigráfico analizado, los ejemplares de este trabajo proceden del banco anteriormente descrito (muestras P-840 y 99033108 a 99033111), pues es el único que nos ha permitido obtener ejemplares completos y alguno de ellos aislado, en un estado de conservación excepcional al mostrar con detalle todos los elementos de la estructura y microestructura de la concha. La ausencia de lavado y removilización del sedimento permitió que los individuos se conservaran bastante completos y en posición de vida. Asimismo, los procesos de dolomitización temprana afectaron de manera intensa a la matriz micrítica, pero no a las conchas de los rudistas, lo que por un lado permitió mantener las características estructurales de éstas y por otro, contribuyó a dar una rápida consistencia inicial al sedimento, lo que nos ha facilitado la recogida de material compacto (Fig. 3e,f) para su posterior estudio en laboratorio. Se ha considerado también un ejemplar de la muestra 99033115 , procedente de un banco de removilización y acumulación de fragmentos, situado dentro del tramo margoso-carbonatado superior. Los intentos realizados en otros niveles resultaron infructuosos por el elevado grado de fragmentación de las conchas y la fácil disgregación de las muestras.

Familia Radiolitidae d'Orbigny, 1847

nom. correct. Gray, 1848 (ex Radiolidae d'Orbigny, 1847)

Género Bournonia Fischer, 1887

Especie tipo: Sphaerulites Bournonii des Moulins, 1826, p. 124; por designación original de Fischer 1887, p. 1067.

Se conocen más de 20 especies del género Bournonia, aunque la descripción de la mayoría de ellas es insuficiente, especialmente con referencia a los caracteres internos y a la microestructura de la concha. La revisión de las especies del género se hace imprescindible antes de abordar cualquier intento de filogenia del grupo.

\section{Bournonia gardonica (Toucas, 1907)}

Figs. 3b, e-g; 4a-e; 5a-e; 6a-d

1907 Agria gardonica Toucas, 25, lám. 2, figs. 6-10.

1910 Bournonia gardonica (Toucas); Douvillé, 49.

1926 Bournonia gardonica (Toucas); Parona, 34.

Material: Los ejemplares, predominantemente valvas derechas, se encuentran incluidos en una matriz calcárea, esporádicamente en contacto uno con otro y es difícil obtener ejemplares aislados completos. Los bloques calcáreos se han registrado como PUAB-43.930 a 43.948, 99033108 a 99033111 y 99033115 . De estos últimos bloques se han hecho dos láminas delgadas de cada uno, que se registraron aparte, respectivamente 33815 a a 33818 b y 33822 a y b. Todo el material descrito y figurado se encuentra depositado en las Colecciones de Paleontología de la Universidad Autónoma de Barcelona.

\section{Descripción}

Los ejemplares son cilindro-cónicos, tienen entre 1 y 3 $\mathrm{cm}$ de diámetro máximo y hasta $10 \mathrm{~cm}$ de longitud. La superficie externa de la valva derecha (valva inferior o fija) es bastante lisa, presenta únicamente dos bandas radiales salientes, la anterior más ancha y cóncava, y pocas costillas radiales redondeadas.

La sección transversal de la valva derecha (inferior o fija) muestra la ausencia de cresta ligamentaria y la estructura de las bandas radiales (Figs. 4, 5). Éstas corresponden a pliegues planos hacia abajo y hacia fuera de las láminas; el pliegue anterior es más ancho que el posterior y está ligeramente desdoblado por lo que resulta cóncavo en el centro. La interbanda está reducida al surco que separa las dos bandas y corresponde a un pliegue agudo hacia arriba y hacia dentro de las láminas. En ejemplares juveniles (Fig. $3 \mathrm{~g}$ ), o en los primeros estadios de crecimiento en ejemplares adultos, las dos bandas están muy juntas y la interbanda es estrecha; en ejemplares adultos y sin restricciones de espacio, pueden estar separadas por una interbanda bastante más ancha y ocupar un ángulo mayor del perímetro.

El estudio de secciones transversales, longitudinales, radiales, tangenciales y oblicuas permite reconocer y describir la estructura de las láminas que constituyen la concha externa calcítica prismática. En sección radial, puede observarse que las láminas están muy inclinadas, por lo que la concha es relativamente delgada, llega a alcanzar raramente $5 \mathrm{~mm}$ de espesor para una concha de $3 \mathrm{~cm}$ de diámetro. En el margen interno de la concha presentan una inflexión (Figs. 6b,c) que provoca el surco que rodea el margen interno de la concha en la comisura (Fig. 5e).

La estructura calcítica prismática de las láminas o es compacta o presenta crestas radiales originadas por repliegues en las láminas. Puede observarse la transición de estructura compacta a estructura con crestas radiales dentro de una misma lámina o el predominio de una u otra estructura en distintas partes de la concha. Es de señalar que 

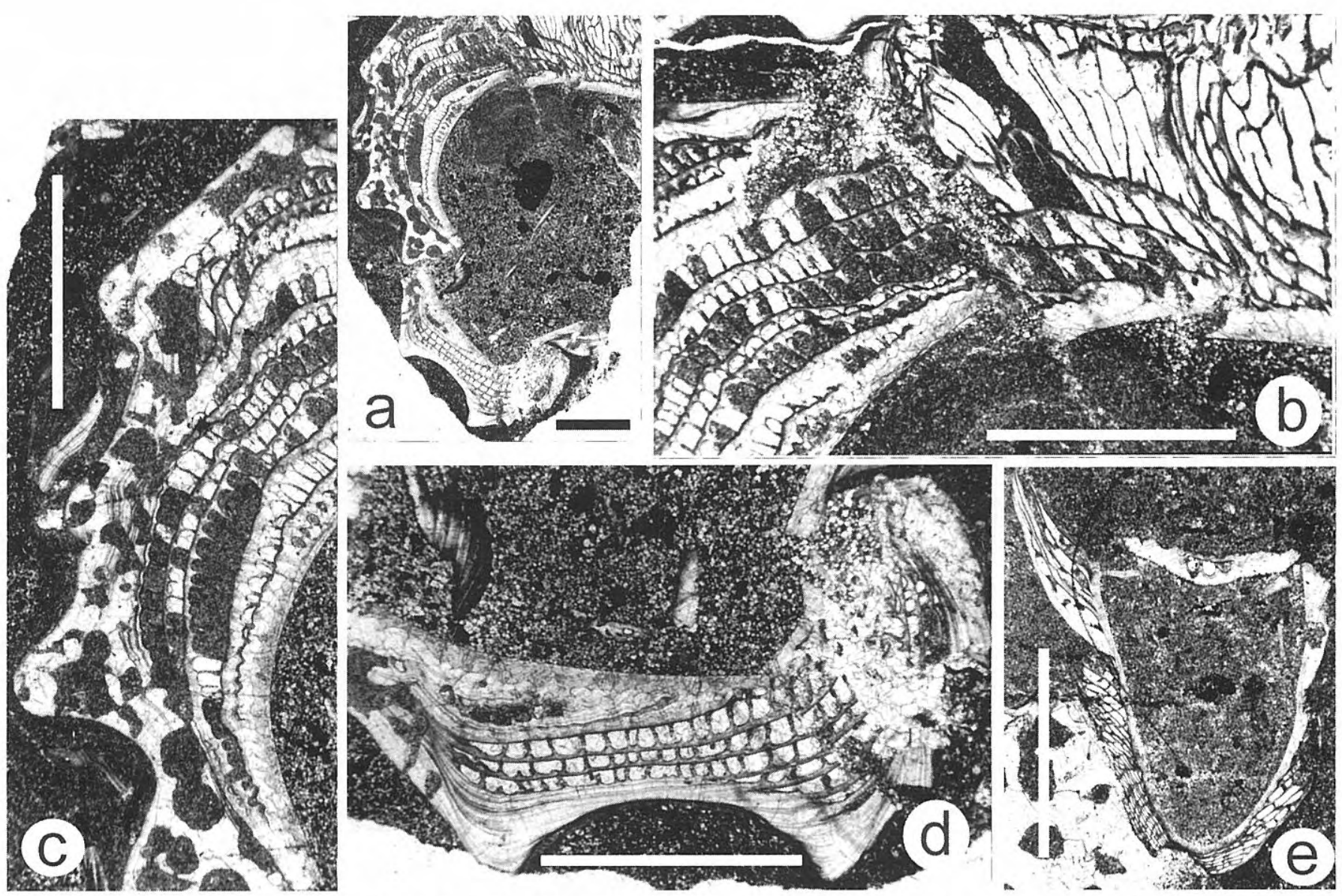

Figura 5. Bournonia gardonica (Toucas). a. Sección oblicua en lámina delgada de la valva inferior (derecha) de un ejemplar de la muestra 99033110 (lámina 33.817b). La sección es bastante paralela a la inclinación de las láminas en la parte superior derecha de la fotografía (lado dorsal) y casi perpendicular a las mismas en la parte inferior izquierda (banda anterior en el lado ventral). b. Detalle del lado anterodorsal. Las crestas en la superficie superior de las láminas son radiales y no se cierran para formar celdas. c. Detalle del lado anterior. Se cortan varias láminas que presentan crestas. Las láminas con estructura compacta están bioperforadas. La ornamentación externa de la concha consta de ligeras costillas originadas por ondulaciones de las láminas. d. Detalle de la banda anterior. Se evidencia que ésta corresponde a un pliegue plano hacia abajo y hacia fuera de las láminas, el desdoblamiento del pliegue dando, en este caso, una exagerada concavidad de la banda, y la diferencia en la proporción láminas compactas/láminas con crestas con respecto a la sección de la banda en la figura 4e. e. Sección longitudinal en lámina delgada de un ejemplar juvenil de la misma muestra (lámina 33.817a). Se observa el surco que rodea el margen interno de la concha en la comisura en la valva inferior (derecha) y que la valva superior (izquierda) es cóncava y presenta la misma estructura de láminas compactas o con crestas, con microestructura prismática simple en ambos casos, que la valva inferior (derecha). La barra de escala corresponde a $5 \mathrm{~mm}$ en todas las fotografías. a. Oblique thin section of the lower (right) valve of one specimen from sample 99033110 (thin section 33.817b). The section is nearly parallel to the inclination of the laminae, in the right upper part of the photograph (dorsal side) and nearly perpendicular to them in the left lower part (anterior band at the ventral side) b. Detail of the anterodorsal side. Crests at the upper surface of the laminae are radial and not joined as to form cells. c. Detail of the anterior side. Several laminae with crests are cut. Laminae with compact structure are bored. Slight ribs formed by waves of the laminae constitute the external shell ornamentation. d. Detail of the anterior band. It is evident that it corresponds to an out-and down-fold of the laminae, the bending of the fold responsible, in this case, for an exaggerated concavity of the band and the difference in compact laminae/laminae with crests ratio compared with the band section in figure 4e. e. Longitudinal thin section of a juvenile specimen from the same sample (thin section 33.817a). It is evident the sulcus surrounding the lower (right) valve's inner shell margin at the commissure as well as that the upper (left) valve is concave and it has the same structure as the lower (right) valve, laminae compact or with crests, with simple prismatic microstructure in both cases. Scale bar corresponds to $5 \mathrm{~mm}$ in all photographs.

únicamente la parte con estructura compacta aparece con bioperforaciones.

Como la sección transversal (Figs. 4, 5), al igual que ocurre en la sección tangencial (Fig. 6b), corta oblicuamente sucesivas láminas, puede aparecer un modelo cuadrangular por combinación de láminas y crestas radiales, que en 

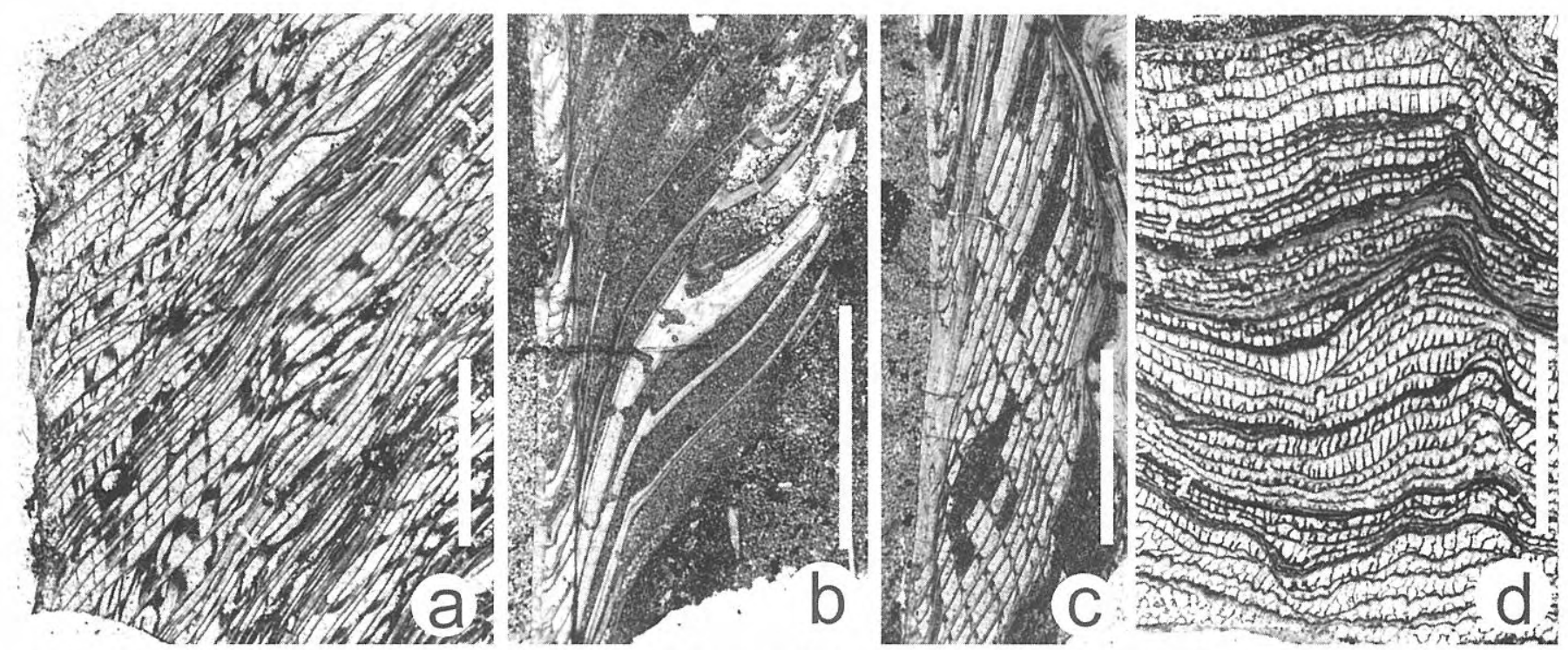

Figura 6. Bournonia gardonica (Toucas). a. Sección casi radial en lámina delgada de la valva inferior (derecha) de un ejemplar de la muestra 99033115 (lámina 33.822a). El margen interno de la concha está situado en la parte izquierda de la fotografía. Aparecen paquetes de láminas en los que no se corta ninguna cresta radial y otros en los que se cortan oblicuamente. b. Sección radial en lámina delgada de la valva inferior (derecha) de un ejemplar de la muestra 99033109 (lámina 33.816a). El margen interno de la concha está situado en la parte izquierda de la fotografía. Se observa la ausencia de estructura celular y la inflexión de las láminas junto al margen interno de la concha, responsable del surco que rodea este margen interno en la comisura y del aspecto particular que aparece en esta posición en sección transversal. c. Sección casi radial en lámina delgada de la valva inferior (derecha) de un ejemplar de la muestra 99033111 (lámina 33.818a). El margen interno de la concha está situado en la parte izquierda de la fotografía. Se observa la gran inclinación de las láminas, así como la inflexión hacia abajo de las mismas junto al margen interno de la concha. Únicamente en la mitad inferior de la fotografía, donde la sección es más oblicua, se cortan oblicuamente las crestas radiales de cada una de las láminas, aparentando estructura celular. d. Sección tangencial oblicua en lámina delgada de un ejemplar de la muestra 99033115 (lámina 33.822b). El margen externo de la concha está situado en la parte superior de la fotografía y el interno en la parte inferior. La sección corta muchas láminas, casi perpendicularmente a las mismas, y también las crestas radiales, dando una falsa impresión de estructura celular. La barra de escala corresponde a $5 \mathrm{~mm}$ en todas las fotografías.

a. Nearly radial thin section at the lower (right) valve of one specimen from sample 99033115 (thin section 33.822a). Inner shell margin at the left side. Laminae sets where any radial crest is cut and others in which they are cut obliquely, are evident. b. Radial thin section of the lower (right) valve of one specimen from sample 99033109 (thin section 33.816a). Inner shell margin at the left side. The absence of any cellular structure and the inflexion of the laminae close to the inner shell margin, responsible for the sulcus which surrounds this inner margin at the commissure and for the peculiar aspect there at transverse section, are evident. c. Nearly radial thin section of the lower (right) valve of one specimen from sample 99033111 (thin section 33.818a). Inner shell margin at the left side. High angle inclination of the laminae, as well as their down-inflexion close to the inner shell margin, are evident. Radial crests of each lamina are obliquely cut only at the lower half of the photograph, where the section is more oblique, resembling cellular structure. d. Oblique tangential thin section of one specimen from sample 99033115 (thin section 33.822b). Outer shell margin at the upper side and inner shell margin at the lower side. Many laminae, as well as the radial crests, are nearly perpendicularly cut, giving a false look of cellular structure. Scale bar corresponds to $5 \mathrm{~mm}$ in all photographs.

ningún caso debe confundirse con una estructura celular. La sección radial (Figs. 6a-c) corta o bien únicamente las láminas (Fig. 6b) o también, oblicuamente, las crestas radiales (Fig. 6a,c).

La valva izquierda (valva superior o libre) ha sido observada únicamente en la sección longitudinal de un ejemplar juvenil conservado en el sedimento de relleno de un ejemplar adulto (Fig. 5e), es plano-cóncava, adaptándose a la dirección de crecimiento de las láminas de la valva derecha y presenta la misma estructura en láminas compactas o con crestas radiales.

\section{Discusión}

La estructura y disposición de las láminas que forman la concha, la ausencia de cresta ligamentaria y la localización de las bandas radiales en pliegues, son caracteres diagnósticos del género Bournonia; así como el desarrollo de estas últimas y especialmente el hecho de que la anterior sea cóncava por desdoblamiento del pliegue permiten atribuir los ejemplares a la especie $B$. gardonica. La estructura de las láminas en el género Bournonia está descrita en Mohanti y Pons (1999), y la interpretación correcta de las bandas radiales del mismo 
ya se encuentra en Douvillé (1910). Este último autor reconoció la adscripción al género Bournonia de la especie gardonica y, al compararla con su especie $B$. fortaui, remarca el espacio considerable ocupado por las dos bandas en el holotipo de Toucas (1907, lám. 2, figs. 2, 2a); tal como se ha argumentado en el apartado anterior, esto depende del estadio de crecimiento y disponibilidad de espacio y puede observarse en otro ejemplar juvenil de la misma localidad figurado por Toucas (1907, lám. 2, figs. 8, 8a) y perteneciente a la colección de la Escuela de Minas de París, que se conserva en Lyón.

Esta especie fue descrita y figurada por primera vez por Toucas (1907), como Agria gardonica, del Coniaciense de Gattigues (Gard, Francia), junto a ejemplares de otras localidades del sur de Francia (Piolenc, Vaucluse y Beausset, Var), señalando también su presencia en el Coniaciense de Rochefort (Landes). Parona (1926) la mencionó de calizas con miliólidos entre Pisino y Vermo (Istria, Croacia). Cestari y Pons (en prensa) señalan su presencia en el Cilento (sur de Italia), en una asociación de rudistas y foraminíferos en facies someras de plataforma característica del Coniaciense y correspondiente al evento K de Cestari y Sartorio (1995) en el Dominio Periadriático.

\section{CONCLUSIONES}

La Formación Calizas de Hortezuelos, en la sección del Barranco de las Cuevas, está constituida por una sucesión de calizas bioclásticas y micríticas, poco dolomitizadas y con rudistas bien conservados, que presentan dos tramos margoso-carbonatados intercalados, definiendo varios conjuntos de estratos.

El análisis de facies del conjunto bioclástico superior permite reconocer tres términos principales y un subtérmino, cuya asociación muestra un patrón de apilamiento sedimentario formado por secuencias de somerización perimareales de una plataforma carbonatada.

Los rudistas pertenecen a la especie Bournonia gardonica (Toucas, 1907) y, gracias a su conservación, se ha podido ampliar la descripción original de la especie, especialmente en la estructura de la concha.

El hecho de ser esta especie característica del Coniaciense, permite atribuir esta edad a la Formación Calizas de Hortezuelos, resultando la primera datación precisa de la unidad carbonatada superior del Cretácico en todo el borde meridional del Sistema Central.

Esta datación confirma en parte el modelo cronoestratigráfico secuencial propuesto con anterioridad para el Cretácico del borde meridional del Sistema Central.

\section{AGRADECIMIENTOS}

Este trabajo ha sido realizado en el marco de los proyectos de investigación de la DIGICYT PB96-0676 y
PB98-0876. El manuscrito original se elaboró durante una estancia de J.G. en el Departamento de Geología de la Universidad Autónoma de Barcelona, subvencionada por el "Programa 2001 de Ayudas para Estancias de Investigación" del Consejo Social de la Universidad de Alcalá. A. Prieur y M. Sirvent (Département des Sciences de la Terre, Université de Lyon I) facilitaron a J.M.P. el acceso a las colecciones a su cargo. Las observaciones de los revisores P. Skelton y M. Floquet, y de los editores M.L. Martínez y S. Fernández, ayudaron a mejorar el texto original. Nuestro agradecimiento a todas estas instituciones y personas.

\section{BIBLIOGRAFÍA}

Almendros, G., Álvarez Ramis, C. y Polo, A. 1982. Estudio paleobioquímico de la materia orgánica de restos fosilizados de Frenelopsis procedentes del supracretácico de Torrelaguna (Madrid). Revista de la Real Academia de Ciencias Exactas Físicas y Naturales, 76-2, 285-302.

Alonso, A. y Mas, R. 1982. Correlación y evolución paleogeográfica del Cretácico al norte y al sur del Sistema Central. Cuadernos de Geología. Ibérica, 8 , 145-166.

Álvarez Ramis, C. y Doubinger, J. 1980. Los microfósiles vegetales del Cretácico superior de Torrelaguna. Boletín de la Real Sociedad Española de Historia Natural (Sección Geológica), 78, 205-215.

Álvarez Ramis, C. y Fernández Marrón, M.T. 1990. Palinomorfos atribuidos a Gimnospermas hallados en niveles supracretácicos del embalse de El Vellón (Provincia de Madrid). Actas del VII Simposio de Palinología, 437-443.

Álvarez Ramis, C., Clemente Belmonte, P., Fernández Marrón, M.T., Galán Ruiz, C. y Gómez Porter, P. 1987. Formas de Normapolenes del Senoniense del embalse del Vellón (Madrid). Resúmenes de la VIII Bienal de la Real Sociedad Española de Historia Natural (Sección Geológica), 205-215.

Álvarez Ramis, C., Fernández Marrón, M.T. y Gómez Porter, P. 1992. Presencia de restos de Glyptostrobus próxima a la especie actual $G$. lineatus (Poiret) Druce en niveles terminales de la serie supracretácica del borde sur de la Sierra de Guadarrama, Barranco de Patones (Madrid). Actas III Congreso Geológico de España, 1, 483-486.

Álvarez Ramis, C., Clemente Belmonte, P. y Fernández Marrón, M.T. 1996. Avance del estudio paleopolínico de angiospermas procedente de la formación cretácica del Barranco de las Cuevas (Madrid). Geogaceta, 20, 201203.

Bellido, F., Casquet, C., González-Lodeiro, F., Martín, M., Martínez, J., Navidad, M., y del Olmo, A. 1987. Memoria de la Hoja 509 (Torrelaguna) del Mapa Geológico de España a escala 1:50.000, $2^{a}$ Serie. Instituto Tecnológico y Geominero de España, Madrid,130 pp.

Bellido, F., Escuder, J., Klein, A., del Olmo, A., Casquet, C., Navidad, M. y Peinado, M. 1988. Memoria de la Hoja 485 (Valdepeñas de la Sierra) del Mapa Geológico de 
España a escala 1:50.000, $2^{a}$ Serie. Instituto Tecnológico y Geominero de España, Madrid, 84 pp.

Cestari, R. and Pons, J.M. (in press). Coniacian-Santonian rudist facies in Cilento (southern Italy). Courrier Forschungsinstitut Senckenberg,

Cestari, R. and Sartorio, D. 1995. Rudists and facies of the Periadriatic Domain. Agip S.p.A., S. Donato Milanese, 207 pp.

Concha, S. de la 1953. Memoria de la Hoja 485 (Valdepeñas de la Sierra) del Mapa Geológico de España a escala 1:50.000, $1^{a}$ Serie. Instituto Geológico y Minero de España, Madrid, 87 pp.

Corchón, F. 1976. Estudio hidrogeológico del Cretácico de los alrededores de Torrelaguna (Madrid y Guadalajara). Boletín del Servicio Geológico de Obras Públicas, 40, $189 \mathrm{pp}$.

Diéguez, M.C. 1986. Nuevo yacimiento de flora albense en la vertiente sur de la Sierra de Guadarrama. Soto del Real (Madrid). Estudios Geológicos, 42, 361-364.

Diéguez, M.C., Montero, A. y Barrón, E. 1993. Las floras fósiles de la Comunidad de Madrid. In: Madrid antes del hombre. Museo Nacional de Ciencias Naturales y Comunidad de Madrid, 15-20.

Douvillé, H. 1910. Êtudes sur les Rudistes. Rudistes de Sicile, d'Algérie, d'Egypte, du Liban et de la Perse. Mémoires de la Societé géologique de France, Paléontologie, 41, 84 pp., 7 lám.

Fischer, P. 1887. Manuel de Conchyliologie et de Paléontologie conchyliologique ou Histoire Naturelle des Mollusques vivants et fossiles. Savy, Paris, 24+1369 pp.

Fuster, J.M. y Febrel, T. 1958. Memoria de la Hoja 509 (Torrelaguna) del Mapa Geológico de España a escala 1:50.000, $1^{a}$ Serie. Instituto Geológico y Minero de España, Madrid, 102 pp.

García, A., Gil, J. y Segura, M. 1996. La terminación sobre el Macizo Hespérico de las plataformas sedimentarias cretácicas del Surco Ibérico, a lo largo del flanco meridional de la Cordillera Carpetana (Guadalajara y Madrid). In: Itinerarios Geológicos desde Alcalá (Eds. M. Segura, I. Bustamante y T. Bardají). Universidad de Alcalá, 247-269.

Gil, J. y García, A. 1996. El Cretácico del borde meridional del Sistema Central; Unidades Litoestratigráficas y secuencias deposicionales. Estudios Geológicos, 52, 37 49.

Gil, J., García-Hidalgo, J.F., Segura, M. e Iglesias, J.A. 2000. Estratigrafía del Cretácico en el sondeo del Sifón de los Yesos (Torrelaguna) y su correlación con los afloramientos de superficie. Estudios Geológicos, 56, 19 29.

Gómez Porter, P. 1984. Nota sobre la macroflora del Cretácico superior de Embalse del Vellón. Colecciones de Paleontología, 39, 61-64.

Gray, I.E. 1848. On the arrangement of the Brachiopoda. Annual Magazine of Natural History (2), 2, 435-440.

Haq, B.U., Handerbol, J. and Vail, P.R. 1988. Mesozoic and Cenozoic chronostratigraphy and cycles of sea level change. In: Sea level changes. An integrated approach (Eds. C. K. Wilgus, B. S. Hastings, C.G.S.C. Kendall, H.W. Posamentier, C.A. Ross and J.C. Van Wagoner), SEPM (Society for Sedimentary Geology), Special Publication, 42, 71-78.

Mohanti, M. and Pons, J.M. 1999. The rudist Bournonia mutabilis (Stoliczka, 1871): redescription based on new material from the Ottakkovil Formation (Maastrichtian), Ariyalur Region, South India. Geobios, 32, 853-859.

Moulins, Ch. des 1826. Essai sur les Sphérulites qui existen dans les collections de MM. F. Jouanet et Ch. des Moulins et considérations sur la famille à laquelle ces fossiles appartiennent. Bulletin d'Histoire naturelle de la Societé Linnéenne de Bordeaux, 1, 148-303.

Orbigny, A. d' 1847. Considérations zoologiques et géologiques sur les Brachiopodes ou Palliobranches (parts 1-2). Comptes Rendus Hebdomadaires des Séances de l'Académie des Sciénces, 25 (5), 193-195; 25 (7), 266-269.

Parona, C.F. 1926. Ricerche sulle rudiste e su altri fossili del Cretacico superiore del Carso Goriziano e dell'Istria. Memorie dell'Istituto Geologico della $R$. Università di Padova, 7, 1-56, 6 lám.

Portero, J.M., Díaz Molina, M., González Lodeiro, F., Pérez González, A., Gallardo, J., Aguilar, M.J. y Leal, M.C. 1990. Memoria de la Hoja 485 (Valdepeñas de la Sierra) del Mapa Geológico de España a escala 1:50.000, $2^{a}$ Serie. Instituto Tecnológico y Geominero de España, Madrid, 84 pp.

Prado, C. de 1864. Descripción física y geológica de la provincia de Madrid. Junta General de Estadística, Madrid, 219 pp.

Toucas, A. 1907. Études sur la classification et l'évolution des Radiolitidés. Mémoires de la Société Géologique de France. Paléontologie, 36, 132 pp., 24 lám., 3 tab.

Vera, J.A., Riba, O. y Reguant, S. 1989. Glosario de términos relacionados con el análisis de cuencas. Revista de la Sociedad Geológica de España, 2, 375-394. 